\title{
Multilayer optics for hard x-ray astronomy
}

Suzanne E. Romaine, Adrian Ivan, Ricardo J. Bruni, Finn Erland Christensen, Fiona A. Harrison, et al.

Suzanne E. Romaine, Adrian Ivan, Ricardo J. Bruni, Finn Erland Christensen, Fiona A. Harrison, William W. Craig, Paul Gorenstein, "Multilayer optics for hard x-ray astronomy," Proc. SPIE 4138, X-Ray Optics, Instruments, and Missions IV, (28 November 2000); doi: 10.1117/12.407551

SPIE Event: International Symposium on Optical Science and Technology, 2000, San Diego, CA, United States 


\title{
Multilayer Optics for Hard X-ray Astronomy
}

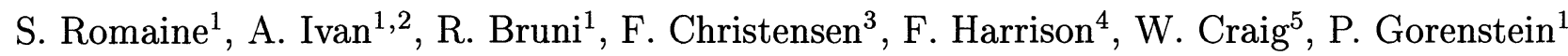 \\ ${ }^{1}$ Harvard-Smithsonian Center for Astrophysics \\ 60 Garden Street, Cambridge, MA 02138 \\ ${ }^{2}$ Massachusetts Institute of Technology \\ Mass Ave, Cambridge, MA 02138 \\ ${ }^{3}$ Danish Space Research Institute \\ Juliane Maries Vej 30, Copenhagen, DK-2100 \\ ${ }^{4}$ California Institute of Technology \\ Pasadena, CA 91125 \\ ${ }^{5}$ Columbia Astrophysics Laboratory \\ 136 S. Broadway, Irvington, NY 10533
}

\begin{abstract}
We are engaged in a program to develop focusing hard X-ray optics for future X-ray astronomy missions (such as the Hard X-ray Telescope of Constellation-X) and have built a DC magnetron sputtering system to deposit multilayers on candidate substrates for future telescopes. Although our emphasis is on the multilayer coating of integral cylindrical optics which will provide the highest spatial resolution, other types of substrates can easily be coated in this system. We present specular reflectivity data (using $C u K_{\alpha} \mathrm{X}$-rays) of W/Si constant $d$ and depth graded-d multilayer depositions on substrates such as thermally formed DESAG glass and Duran glass cylinders. We will present data to show both azimuthal and linear uniformity of these coatings.
\end{abstract}

Keywords: X-ray Telescopes, Multilayers,X-ray optics

\section{INTRODUCTION}

We are involved in a collaboration to develop technology for the Hard X-ray Telescope (HXT) optics of Constellation-X [1]. In particular, we have built a coating chamber to fabricate uniform multilayer coatings on large area Wolter type optics. We have discussed in previous work [2] the development of our coating system; in this paper we present a report of some of our recent uniformity measurements.

The system was designed to provide uniform coatings on the inside surface of integral Wolter-type X-ray optics. The flexibility of this system provides for the capability of uniformly coating segmented as well as 
integral optics. The data presented in this paper is based on the coating of thermally formed DESAG glass segmented optics.

\section{EXPERIMENTAL DETAILS}

The substrates used for the uniformity tests reported here were thermally formed segmented slump glass pieces [3]. Each segmented substrate was $20 \mathrm{~cm}$ in height and slumped to form a 120 degree segment of a conical Wolter-I type optic with a radius of approximately $11 \mathrm{~cm}$. Three of these segments were mounted in the chamber to form a 'complete optical element' as shown in figure 1. Below the center of each substrate a 2 inch silicon wafer was mounted to act as a witness sample for the coating run. The response of the silicon wafers is known from previous tests, therefore the wafers can be characterized to provide details of the coating run. Any deviation between the response of the silicon wafer and the response of the slump glass substrate can then be attributed to the difference in the surface properties of the substrates (such as surface microroughness). After coating, the substrates were removed from the chamber and $8 \mathrm{kev}$ specular reflectivity data was taken using the X-ray reflectivity (XRR) system shown in figure 2. The IMD software

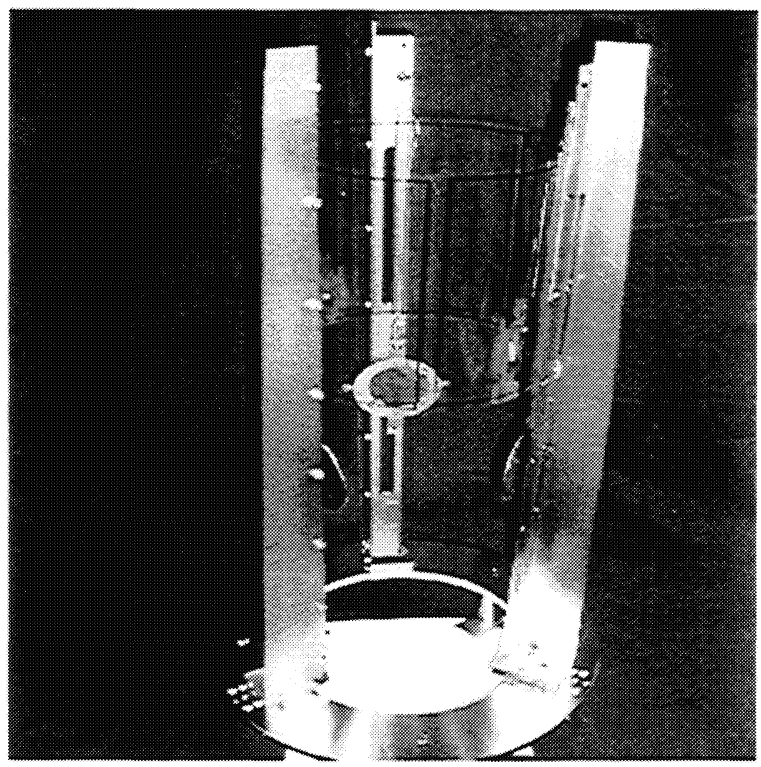

Figure 1: Second generation sample holder showing three 120 degree segments of slump glass mounted for coating run (a sharpie pen was used to outline the slumped glass for better definition in this photograph). Also shown here are three silicon wafers which will be used as witness samples for the coating run.

package [4] was used to model the data to extract a d-spacing, interface microroughness and gamma from the specular scan ( $\mathrm{N}$, the number of bilayers, was fixed at 60 for the run).

To look at the uniformity of the coating, specular reflectivity scans were taken at several different points on each substrate. The schematic of one piece of slump glass in figure 3 shows the approximate positions where the reflectivity data was taken on each substrate: the circles mark the 16 scan positions for S1, the squares mark the 6 positions for S2 and the X's mark the 15 positions for S3. (Although 16 points were scanned for S2, only 6 scans were analyzed before this conference.) On any one substrate the area sampled covered up to 90 degrees in azimuth and up to $15 \mathrm{~cm}$ in length. A tantalum slit was used to define the width of the beam impinging on the sample. The slits were fixed so that the footprint of the beam on the sample increased as the graze angle decreased. The slit was set such that the footprint of the beam was $50 \mathrm{~mm}$ at a grazing angle of 0.2 degrees. In this way several measurements could be taken along the longitudinal 


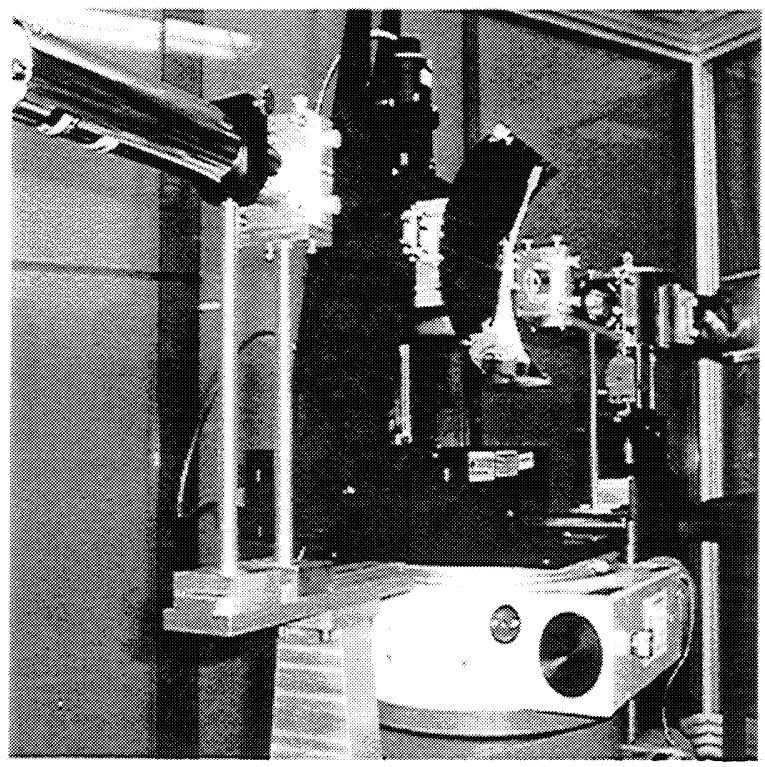

Figure 2: X-ray reflectivity system which has been upgraded this past year to provide additional computer controlled degrees of freedom in sample motion. Shown here is one of the coated slump glass segments in the XRR sample holder. The scintillator detector can be seen in the upper left of the photograph.

axis of the face of the sample, scanning a different area of film for each measurement. The results are then compared to provide a measurement of uniformity across the coated segment. By comparing data from all 3 segments, we can estimate the uniformity of the coating of a complete ( 360 degree) optic.

Based on data collected from several previous test runs of W/Si multilayers, coating parameters were set to yield: $\mathrm{N}=60, \mathrm{~d}=29.0, \gamma=0.4$. An interface 'roughness' of $\approx 3-4 \AA$ was expected for the silicon wafers; this run provides our first data for microroughness on the slumped glass segments.

\section{$3 \quad$ RESULTS and DISCUSSION}

\subsection{Substrates}

The slump glass segments coated were taken from a batch of test substrates and therefore did not have 'perfectly slumped' figures. Any deviation in substrate surface form will create a difference in targetsubstrate distance (as the optic rotates around the target during the coating process) which will translate into a difference in coating thickness, or d-spacing (i.e. uniformity). Because it's difficult to exactly quantify the skewness in the substrates, and because this is not an issue for the flight glass, we did not correct for this contribution and simply note that results reported here for uniformity will improve with flight quality optics. In addition, the optic was a conical approximation to a Wolter optic and the radius differed by $\approx$ $3 \%$ from one end to the other. It is straight forward to accommodate the coating for this gradual change in radius by mounting the optic on a slight angle during coating, but this was not done for the optic discussed here. Therefore a $3 \%$ difference in d-spacing from one end to the other was expected. 


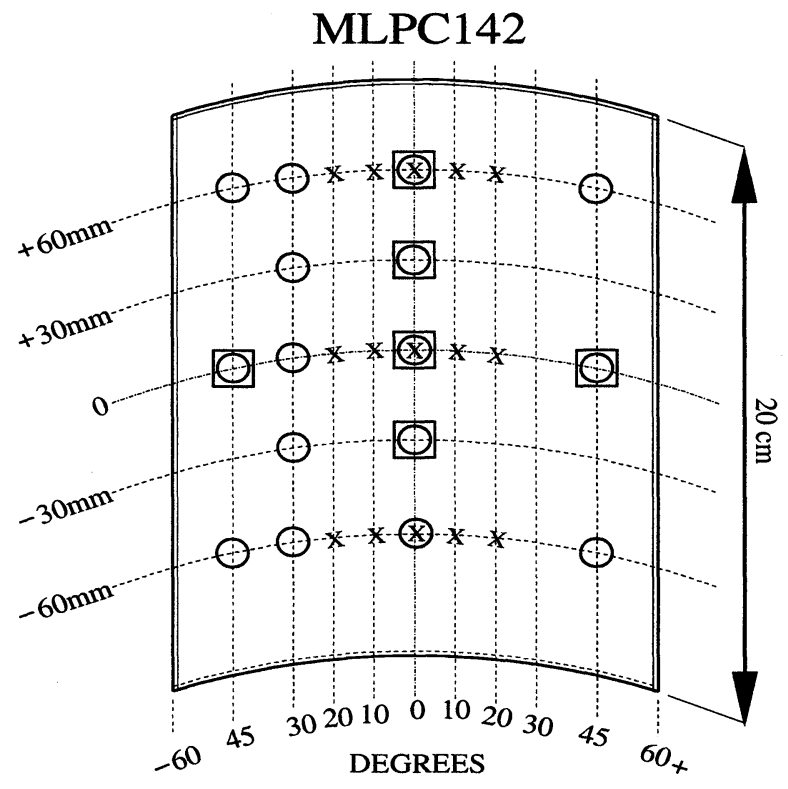

Figure 3: Schematic of thermally formed slump glass substrates used for coating uniformity tests. Each substrate was shaped to form a 120 degree segment of a conical Wolter-I optic. The symbols mark the positions on the substrate where the XRR scans were taken. A different symbol is used for each of the three substrates coated: circles indicate positions for scans for sample S1, squares for sample S2 and X's for sample S3. All samples are from coating run 142. See text for more details.

\subsection{Reflectivity Scans}

Data for representative specular scans from the coating run is shown in figure 4, where the data is represented by triangles and the model fit is given by the solid line. All scans were taken to 4 degrees in theta which, for the parameters used, includes 2 Bragg peaks. The plot in figure 4a is representative of the scans from the slump glass optics, the plot in figure $4 \mathrm{~b}$ is representative of the scans for the silicon wafers coated in the same coating run. The silicon wafers have an interface roughness of 3 to $3.5 \AA$ (which is the same as our previous data for $\mathrm{W} / \mathrm{Si}$ multilayers on silicon wafers) and the slump glass scans show a slightly higher interface roughness of 4 to $4.5 \AA$. This is consistent for all the data in this coating run. The height of the Bragg peaks in the scans from the slumped glass are lower than those of the silicon wafers. This may be coupled with a slight broadening of the peak, but more analysis is needed before we can discuss this in detail.

\subsection{Uniformity}

Any non-uniformity in the coating of the optic will contribute to a reduced effective area [6]. Therefore, it is important to measure the uniformity of our coatings to be certain we are not contributing to losses in effective area. Although graded-d multilayers will be used to coat the Hard X-ray Telescope of Constellation-X, a better probe of uniformity of coating can be done with constant-d multilayers. The sharp well defined bragg peaks in the X-ray specular reflectivity response of constant d-spaced multilayers put a tighter constraint on 

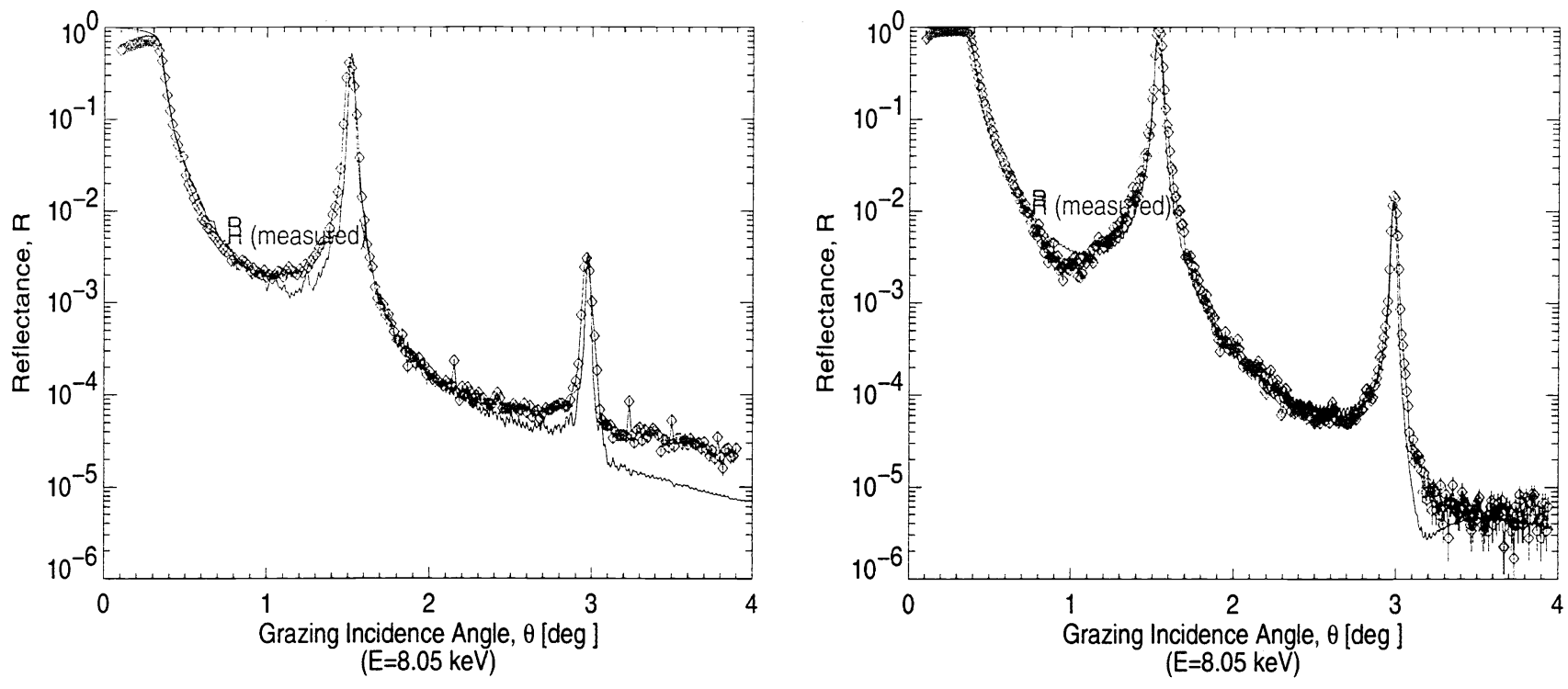

Figure 4: Representative plot of specular reflectivity vs. angle taken at $\mathrm{Cu}-\mathrm{K}_{\alpha}(8.05 \mathrm{keV})$.

(a)data from scan of slumped glass segment

(b) data from scan of silicon wafer witness sample.

the modelled d-spacing than does the response of the graded d-spaced multilayers. Therefore we chose to carry out the uniformity tests using the following coating parameters: $\mathrm{N}=60, \mathrm{~d}=29 \AA, \gamma=0.4$ (where $\gamma$ is defined as reflector thickness over $\mathrm{d}$ ). The $\mathrm{d}$-spacing is determined to $1 \%, \gamma$ to $\approx 10 \%$, the microroughness to better than $0.5 \AA$ and the $\mathrm{N}$ is fixed (known) for a given coating run.

Figure 5 plots the modelled d-spacing data for all three slump glass segments: filled squares for S1 data, open squares for S2 data and X's for S3 data. The data for sample S3 shows a 'trend' in the d-spacing decreasing from upper left to lower right; this was a result of the substrate itself being slightly 'out-of-round' - one side of the substrate was figured to a slightly smaller radius (and therefore higher d-spacing) than the other side. Similarly, substrate S1 (represented by the filled squares) had a skewness in it's figure across the central diagonal which contributed to the pattern of d-spacing that is seen in figure 5 .

The $d$ spacing values for all the data represented here fall within $5 \%$ of the average value of $28.8 \AA$. This is within the acceptable tolerance [6][7] of change in bilayer thickness across the optic without introducing a significant decrease in the throughput or field of view of a telescope such as that currently planned for HXT of Constellation-X. It is expected that these results will be improved by (1) introducing a proper fixture to account for the conical shape of the optics, as discussed above and (2) with the use of flight quality optics as opposed to the test quality discussed here. The analysis of this data is ongoing and a paper is in preparation.

\section{ACKNOWLEDGMENTS}

The software used to model the data was provided by Dr. David Windt of Columbia Astrophysics Laboratory [4]; This work was supported in part by NASA contract NAG5-5095. 


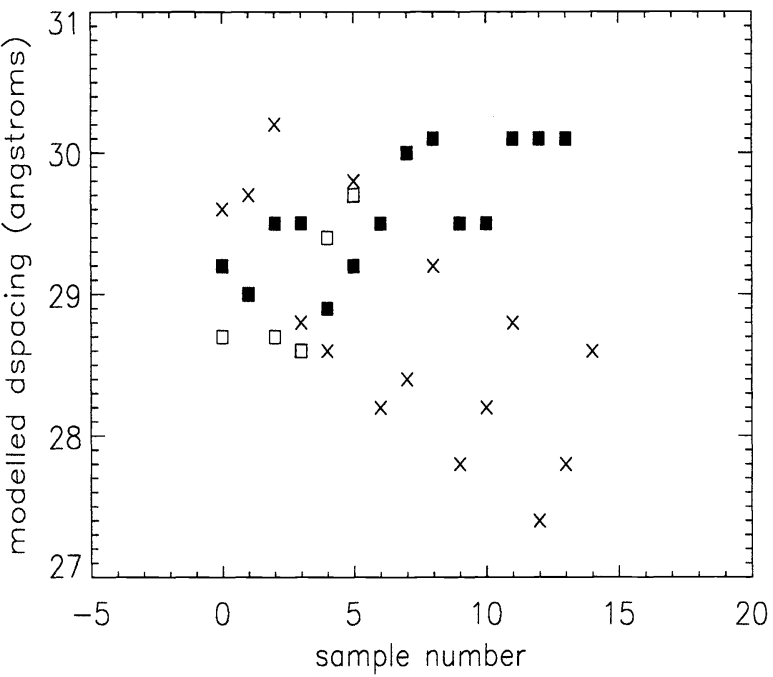

Figure 5: Plot of modelled d-spacing for each reflectivity scan. The data plotted is from 3 different coated segments: filled squares represent data scans from S1, open squares represent data scans from S2 and X's data scans from S3. The mean value of $\mathrm{d}$ for all the points shown is $29.1 \AA$ with a standard deviation of 0.8 $\AA$.

\section{References}

[1] N.E. White and H.D. Tananbaum, "The Constellation X-ray Mission",http://constellation.gsfc.nasa.gov/

[2] S. E. Romaine, et al., "Characterization and Multilayer Coating of Cylindrical X-ray Optics for X-ray Astronomy", Proc. SPIE, 3444, 552-555 (1998).

[3] W.W.Craig et al.,"Hard X-ray optics for the HEFT balloon-borne payload; prototype design and performance", Proc. SPIE, 3445, 1998.

[4] D.L.Windt, "IMD version 4.0", http://www.bell-labs.com / user/windt/idl/imd/index.html

[5] A. Ivan, et al. 1998, "Characterization of graded d-sp acing multilayers for hard X-ray telescopes", Proc. SPIE, this volume.

[6] P.Mao, et al., "Development of grazing incidence multilayer mirrors for hard X-ray focusing telescopes", Proc. SPIE, 3114, 526-534 (1997).

[7] F.E.Christensen, et al., "Hard X-ray characterization of a HEFT single reflection prototype", Proc. SPIE, 4012, 626-638 (2000). 